\title{
Standardised Holographic Patients: An Evaluation of Their Role in Developing Clinical Reasoning Skills
}

\author{
Emma COLLINS $^{\mathrm{a}, 1}$ and Liz DITZEL ${ }^{\mathrm{b}}$ \\ ${ }^{a}$ Principal Lecturer, Otago Polytechnic, Dunedin, New Zealand \\ b Professor, Otago Polytechnic, Dunedin, New Zealand
}

\begin{abstract}
In 2019, two standardised holographic patients viewed through a mixedreality (MR) HoloLens headset were used in a structured learning activity to develop clinical reasoning skills among second-year nursing students ( $\mathrm{N}=99,94 \%)$. Quantitative results indicated that all students felt that being able to closely view holographic patients enhanced their learning experience. Qualitative results showed that the best feature of the MR technology was being able to clinically assess the patient in a safe facilitated environment. Further analysis revealed that students were at the 'beginning' or 'developing' skill level of the vpLCJR. These findings confirm that using standardized holographic patients offers a dynamic and effective experience for students and helps students to develop clinical reasoning and judgement skills. The technology also allows educators to determine a student's development of clinical judgement skills and tailor learning experiences to further develop these skills.
\end{abstract}

Keywords. HoloLens, clinical reasoning, clinical judgment, nursing students

\section{Introduction}

The aim of this research project was to evaluate the efficacy of using standardized holographic patients to assist undergraduate nurses to develop clinical reasoning skills thereby improving quality and safety of patient care. Clinical reasoning is the cognitive process nurses use to collect cues, process information, understand a patient problem or situation, plan and implement interventions, and evaluate and reflect on the care given [1]. Clinical reasoning, however, is not an easy skill to teach students as it takes time to develop and requires clinical nursing situations for them to learn how to do this [2].

The use of Mixed Reality (MR) technology can provide students with safe learning classroom learning opportunities to develop clinical reasoning skills. Microsoft Hololens is a MR device that is worn on the head [3]. Additional information may also be viewed by 'air clicking' a holographic keyboard that displays vital signs (blood pressure, etc.). An application (app); HoloPatient, designed by Pearson (now called GIGXR) is used to provide the holograms. Headset wearers see the hologram and hear breathing sounds via an internal speaker. This app provides educators with access to a virtual standardised patient displaying various symptoms and behaviours that can be downloaded and placed

${ }^{1}$ Corresponding Author, Emma Collins, School of Nursing, Otago Polytechnic, Forth Street, Dunedi, New Zealand; E-mail: Ecollins@op.ac.nz. 
in any environment. The image can be projected anywhere in the room, making it possible to walk around the hologram, gaining a 360-degree view. This allows students to collect visual and aural cues while learning assessment and clinical reasoning skills. Such interactive features greatly enhance realism, reminding learners to look beyond what they first see.

\section{Methods}

During the 2019 academic programme, second-year Bachelor of Nursing (BN) students were invited to participate in a one-hour learning activity designed to develop their clinical reasoning skills. The researchers were trained to use the equipment and technical support was provided to calibrate and set up the equipment for each teaching session.

The study aims were to:

1. Assess the role of using holographic patients in developing nurses' clinical reasoning skills by using the virtual patient version of the Lasater Clinical Judgment Rubric (LCJR).

2. Evaluate the student experience of using HoloLens technology.

The institution's Research Ethics Committee approved this observational study. Students signed consent forms and data were de-identified prior to analyses. Participants were made aware that taking part in this study was voluntary and that worksheets were not part of their course assessment and had no bearing on course grades. Information was collected at the end of each session via pen and paper worksheet by the student. It was then de-identified before being used as data for this project. Worksheets contained openended questions about the overall learning experience and two tables in which students listed patient cues that they observed from the holograms such as 'blue lips', and diagnostic information such as 'poor oxygen perfusion to body organs/cyanosis.

During the learning activity, students wearing the HoLolens headsets viewed two HoloPatients; 'Sandra, a young woman presenting with asthma, and 'Mr Littlefield', a middle-aged man admitted with chest trauma. The nursing scenarios evolve in a series of visual clips.

For this study, students assessed each patient's clinical condition by recording as many cues as possible for all the clips, noting the meaning of each cue in the blank spaces on their worksheet tables. Student worksheet responses were checked for observational accuracy by the researchers. Participants' stage of skill development was then assessed using the Virtual Patient Version of the Lasater Clinical Judgement Rubric (vpLCJR) by the researchers. The researchers independently assessed where the student is on the rubric, using data from the worksheet. The vpLCJR [4] is based on the Lasater Clinical Judgement Rubric and assesses skill development according to four levels: 1=beginning, $2=$ developing, $3=$ accomplished, $4=$ exemplary.

\section{Results}

All $(\mathrm{N}=99)$ participants enjoyed the learning activity and reported that their learning was enhanced though using the HoloLens technology. Table 1 shows a summary of themes identified from responses to the open-ended questions. 
Table 1. Thematic analysis results.

\begin{tabular}{|l|l|}
\hline Item & Themes \\
\hline Student experience and satisfaction & Visualization of the patient \\
& Safe learning environment \\
& Authenticity of the experience \\
& Instant discussion \\
\hline Benefits of using this technology & Safe and stress-free learning environment \\
& Visualization of the patient \\
& Realistic \\
& Interactive \\
\hline Disadvantages of using this technology & Technical issues \\
& Cannot communicate with the patient \\
\hline
\end{tabular}

Students enjoyed this learning activity and appreciated the opportunity to closely examine a patient in a small group with support from their lecturer. This was verified by statements such "I was able to participate in a 'real life' scenario in a safe environment". Others valued the authenticity of the experience and the fact that they could easily discuss and explore issues among each other by noting "It made the scenario much easier to understand compared to just a written one", and affirming that "It was very realistic, I gathered good information about the patients situation". Participants identified a number of benefits of this mode of learning, particularly the ability to safely practice assessment skills were evidenced by the following comment: "Virtual reality [meaning MR] allows us to view scenarios in a non-stressful and safe space". Learners also appreciated the reality for the learning situation and "having the visual understanding of how the patient looks and what they are experiencing". The authenticity of the patients, for example, Mr Littlejohn's discomfort from his seatbelt bruising across his chest, and Sandra's struggling to breathe, enabled students to "see symptoms actually play out rather than having to imagine them". Visualization of the life-sized person projected into the room and "being able to see the patient and their actual diagnosis", was a highlight of using this technology.

Disadvantages related to the fact that students can only hear the patient through audio on the headset and cannot talk with them, voiced though the following remark that it "would be great if you could communicate with patient". The other drawback related to technical issues, i.e. the "tech isn't perfect, runs into a few problems" such as image drop out that were experienced during the session.

Data from the worksheets indicated that the student were at the 'developing' or 'beginning' level of clinical judgment according to the vpLCRJ. This was not surprising as these students in the second year of the $\mathrm{BN}$ degree, and are novice nursing practitioners. This also reassured staff that learners were at an appropriate stage of skill development, with further progress expected in the BN degree.

\section{Discussion}

These findings indicate that holographic patients were valuable in helping students to develop clinical skills in a safe learning environment. Ensuring nursing students are exposed to a range of digital healthcare technologies in their undergraduate years is an essential part of preparing to become Registered Nurses. A variety of web-based, on-line, and software applications 'apps'; including virtual reality, mixed and augmented reality technology; and interactive games are available to enhance student learning [5-7]. 
Mixed reality (MR) is a new and emerging technology in healthcare practice and nursing education. It provides an experience that bridges virtual and real-world situations, thereby supplementing reality [5,6]. MR redefines how people interact with their environment and the Microsoft HoloLens is used in diverse educational contexts, such as medicine and pathology education [7].

Virtual learning environments (VLE) may be a potential answer to providing a low risk, safer and less stressful context for healthcare professionals including nurses [8]. They may also assist in bridging the 'theory-practice' gap that often exists in healthcare education [9]. In virtual environments, the combination of representational fidelity and learner interaction create a sense of presence for learners, considered to be a hallmark of VLEs [10]. This sense of presence enhances learning through immersion and immediacy, i.e. real-time actions and responses are possible with life-like patients [6]. These immersive learning environments are far less stressful than clinical simulations for learners and have the added benefit of high sense of presence [11].

Different forms of technologies can lead to enhanced engagement in the learning activity, especially where there are well-constructed learning outcomes. Virtual and MR technologies also have the capacity to provide real life learning experiences that promote student engagement and meet the learning needs of a diverse student cohort [12]. Augmented and virtual realities can be utilised in multiple healthcare settings to enhance the patient experience and the care that patients receive [5]. However, research directly linking the use of these technologies to enhanced patient outcomes, is sparse.

\section{Conclusion}

These findings suggest that using MR and virtual learning environments are integral to improving the future quality and safety of patient care. Participants enjoyed this interactive learning activity and valued the authentic context of being able to see, hear and assess a holographic patient. According to the VPLCJR, students were at the 'beginning and developing' stage of clinical judgment which was commensurate with them being second-year nurses, reflecting their status as novices with relatively limited clinical nursing experience. Overall, this study's results indicate that the value of using holograms to assist students to develop clinical reasoning skills is that they are safely exposed to learning experiences not replicable outside of clinical or real-world contexts.

\section{Acknowledgements}

Hugh Harlow, Learning and Teaching Specialist, Otago Polytechnic, Dunedin, New Zealand

\section{References}

[1] School of Nursing and Midwifery, Clinical Reasoning, Instructor Resources, University of Newcastle, Australia, 2009.

[2] Levett-Jones T, Hoffman K, Dempsey J et al. The 'five rights' of clinical reasoning: An educational model to enhance nursing students' ability to identify and manage clinically 'at risk' patients. Nurse Education Today, 20 (2010), 515-520. 
[3] Foronda CL, Alfes CM, Dev P, Kleinheksel AJ, Nelson DA, O’Donnell JM, et al. Virtually nursing: emerging technologies in nursing education, Nurse Educator, 42 (2017), 14-17.

[4] Georg C, Karlgren K, Ulfvarson J, Jirwe M, Welin E. A rubric to assess students' clinical reasoning when encountering virtual patients, Journal of Nursing Education, 57 (2018), 408-415.

[5] Ferguson C, Davidson PM, Scott PJ, Jackson D, Hickman LD. Augmented reality, virtual reality and gaming: an integral part of nursing, Contemporary Nurse, 51 (2016), 1-4.

[6] Wu HK, Lee SW, Chang HY, Liang JC. Current status, opportunities and challenges of augmented reality in education, Computers and Education, 62 (2013), 41-49.

[7] Hanna MG, Ahmed I, Nine J, Prajapati S, Pantanowitz L. Augmented reality technology using Microsoft HoloLens, anatomic pathology: Arch Pathol Lad Med, 142 (2018), 638-644.

[8] Fronczek,AE. Nursing theory in virtual care. Nursing Science Quarterly, 32 (2019), 35-38.

[9] King D, Tee S, Falconer L, Angell C, Holley D, Mills A. Virtual health education: Scaling practice to transform student learning, Nurse Education Today, 71 (2018), 7-9.

[10] Fowler C. Virtual reality and learning: Where is the pedagogy? British Journal of Educational Technology, 46, (2015), 412-422.

[11] Mariana FP, Damian ET, Antonio NF, Nuria PA, Joaquin CM, Rafael MR, et al, Comparative study of a simulated incident with multiple victims and immersive virtual reality, Nurse Education Today, 71 (2018), 48-53.

[12] Ewens B, Geale S, Vafeas C, Foxall F, Loessl B, Smyth A, et al. Humanising the curriculum: the role of a virtual world. Journal of Nursing Education and Practice, 6 (2016), 80-88. 